\title{
Análisis del funcionamiento de las secciones que componen el aula virtual de la cátedra de Física-Química Biológica.
}

Review the performance of the sections of the virtual classroom of the Chair of Physical Chemistry Biological.

Fecha de Recepción

02 de agosto de 2012
Analisar o desempenho dos setores da sala de aula virtual da Cátedra de Físico-Química Biológica.

\section{Aceptado para su publicación}

23 de octubre de 2012
Fermando R. Cuzziol

Profesor Titular. Cátedra de Física-Química Biológica. F.O.U.N.N.E.

E-mail: fernandoramirocuzziol@hotmail.com

Miguel Acuña Auxiliar I ra. Categoría. Cátedra de Física-Química Biológica. F.O.U.N.N.E. E-mail: odontoacuna@gmail.com

\section{Viviana Piatti}

JTP. Cátedra de Física-Química Biológica. F.O.U.N.N.E.

E-mail: anedente@hotmail.com

Facultad de Odontologia. Avenida Libertad 5450. Corrientes.

República Argentina.

\section{Resumen}

En el presente artículo se detallan minuciosamente el número de intervenciones en las diferentes secciones del Aula Virtual, y el grado de participación de alumnos y docentes durante el cursado de la asignatura Física-Química Biológica del ciclo lectivo 20II determinando, de este modo cual de ellas es la más utilizada y cual sería la de mayor importancia para el alumno. Para tal fin se utilizaron los registros obtenidos de la sección Administración de la Plataforma de UNNE Virtual.

Los datos obtenidos fueron analizados desde un punto de vista estadístico descriptiva.

Los resultados obtenidos denotaron que la sección mas visitada fue la de actividades con un número de ingresos de 21.604 que corresponde al $24 \%$ y en segundo lugar la de otros documentos con 16.893 ingresos (20\%), lugares donde los alumnos tuvieron a disposición actividades de auto evaluación y material de estudio de la cátedra. También se demostró por medio de los registros un elevado grado de participación en las otras secciones mencionadas en el artículo con un total de 47.288 ingresos (56\%).

El objetivo de de esta publicación fue la de analizar el funcionamiento de las diferentes secciones que componen el Aula Virtual de Física Química Biológica.

\section{Pallabras Claves}

Educación a distancia, Tutor, Tutorías virtuales, plataforma virtual. 


\section{Abstract}

This article details the number of statements carefully in different sections of the Virtual Classroom, and the degree of participation of students and teachers during the course completed the Physical-Chemical Biology of the school year 20II determining, so which one is the most used and what would be the most important for the student. For this purpose we used the records obtained from the Administration section of the Platform for Virtual UNNE. Data were analyzed from a descriptive statistical standpoint. The results denoted that was the most visited section of activities with a number of admissions of 21,604 corresponding to $24 \%$ and secondly the 16,893 other income documents $(20 \%)$, where the students had available activities self-assessment and study material of the chair. It was also shown by the records of a high degree of participation in the other sections mentioned in the article with a total income of $47,288(56 \%)$.

The objective of this publication was to analyze the performance of the different sections that make up the Virtual Classroom Physics Biological Chemistry.

\section{Key Words}

Distance Education, Tutor, Virtual tutorials, Virtual platform.

\section{Resumo}

Este artigo detalha o número de declarações com cuidado em diferentes seções da Sala de Aula Virtual, eo grau de participação de alunos e professores durante o curso concluído de Biologia Físico-Química do ano letivo 20II, que determina, de modo que um é o mais utilizado e qual seria $\circ$ mais importante para $\circ$ aluno. Para este efeito, foram utilizados os registros obtidos a partir da seção Administração da Plataforma de UNNE Virtual. Os dados foram analisados a partir de um ponto de vista estatístico descritivo. Os resultados denotam que foi a seção mais visitada de atividades com um número de admissões de 21.604 correspondente a $24 \%$ e em segundo lugar os 16.893 documentos outros rendimentos $(20 \%)$, onde os alunos tiveram atividades disponíveis auto-avaliação e material de estudo da cadeira. Também foi demonstrado pelos regis- tros de um alto grau de participação de outras secções mencionadas no artigo com uma renda total de 47.288 (56\%).

O objetivo desta publicação foi analisar o desempenho das diferentes secções que compõem a Sala de Aula Virtual Química Física Biológica.

\section{Palavras Chaves}

Educação à Distância, Tutor, Tutoriais virtuais, Plataforma virtual.

\section{Introducción}

La educación superior, al igual que ocurre en otros niveles y ámbitos educativos, está viviendo un periodo de transformación, provocado entre otros factores, por la irrupción de las telecomunicaciones en nuestra sociedad. Las redes de ordenadores, y en especial Internet, han trastocado las formas y modos en que se genera, gestiona y difunde la información y el conocimiento. La institución universitaria como centro de producción y transformación del saber, no puede, obviamente, renunciar a las enormes potencialidades que ofrecen las redes telemáticas como instrumento o recursos destinados a la formación, y en nuestro caso particular al desarrollo de las tutorías virtuales ${ }^{1-2}$.

La integración de las Nuevas Tecnologías de la Información y Comunicación para el aprendizaje de las ciencias permite: presentar los materiales de estudio a través de múltiples medios y canales. Motivar e involucrar a los estudiantes en actividades de aprendizaje significativo. Proporcionar representaciones gráficas de conceptos y modelos abstractos. Mejorar el pensamiento crítico, habilidades y procesos cognitivos.-Posibilitar el uso de la información adquirida para resolver problemas y para explicar los fenómenos del entorno. Permitir el acceso a la investigación científica y el contacto con científicos y base de datos reales. Ofrecer a docentes y estudiantes una plataforma a través de la cual pueden comunicarse con compañeros y colegas de lugares distantes, intercambiar trabajo, desarrollar investigaciones y funcionar como si no hubiera fronteras geográficas. Las nuevas tecnologías de información y comunicación permiten al estudiante ser el principal actor en la construcción de sus conocimientos en base a situaciones diseñadas por el docente ${ }^{3}$. 
Hoy en día, las computadoras aportan al proceso educativo atributos tales como: una disponibilidad en todo momento para resolución de problemas y comprensión de textos. Permiten a los estudiantes mejorar actitudes para ejercicios. También facilitan el bajo costo de acceso de los usuarios a Internet y a la gran cantidad de información y conocimiento que ofrecen al instante ${ }^{4}$. La escuela presencial siempre seguirá siendo necesaria, pero en lugar de una enseñanza de tipo impersonal, dirigida al promedio de capacidades y de necesidades que se dan en un salón de clases, se instauraría Internet como fórmula válida para proporcionar apoyos individualizados, que tomarían en cuenta las necesidades educativas y las capacidades intelectuales de cada persona ${ }^{5}$.

Las tutorías virtuales usadas como herramienta complementaria, mejoran significativamente las calificaciones de las evaluaciones globales de los estudiantes que la utilizan. Adicionalmente, se evidenció un patrón en la frecuencia de participación de los estudiantes, que se relacionaría con la cercanía a fechas evaluativas. Es necesario que las instituciones de educación superior promuevan prácticas educativas innovadoras que favorezcan el aprendizaje 6 .

Los sistemas de educación y formación abiertos y a distancia han dejado de ser sólo una alternativa más de enseñanza para convertirse en un modelo educativo de innovación pedagógica del presente siglo. En la modalidad de educación a distancia, "el aula virtual" se constituye en el nuevo entorno del aprendizaje al convertirse en un poderoso dispositivo de comunicación y de distribución de saberes que, además, ofrece un "espacio" para atender, orientar y evaluar a los participantes. El aula virtual, disponible en Internet las 24 horas del día, ofrece los servicios y funcionalidades necesarias para el aprendizaje a distancia y responde a la necesidad de los docentes y alumnos de una comunicación directa y atención personalizada inmediata o diferida.

Las ventajas de una plataforma son muchas: Reduce notablemente los costos de la formación, no requiere de un espacio físico, elimina desplazamientos de los participantes, amplía notablemente su alcance dando mayores posibilidades a los que se encuentran más alejados de los Centros de formación, permite el acceso a los cursos con total libertad de horarios, proporciona un entorno de aprendizaje y trabajo cooperativos, distribuye la información de forma rápida y precisa a todos los participantes, prepara al educando para competir en el mercado de manera más ágil, rápida y eficiente, convierte la docencia virtual es una opción real de teletrabajo, de complementariedad, sin lugar a dudas, con la formación presencial y con los soportes didácticos ya conocidos ${ }^{7-8}$.

El campus virtual de una organización universitaria se plantea como un proyecto institucional con la meta de facilitar la integración de las nuevas tecnologías de la comunicación en las prácticas docentes del profesorado, estimular el uso de estas nuevas tecnologías por parte del alumnado y extender la oferta formativa de la universidad a otros grupos sociales. La creación y desarrollo de un campus virtual será asumido e impulsado por los órganos de gobierno de la universidad como un proyecto a medio plazo. Esto significa que se planteará como un sistema de innovación no solo tecnológica, sino también pedagógica de la oferta formativa ofrecida por dicha universidad. Op. Cit. I

\section{Desarrollo}

\section{Situación Contextual}

El presente trabajo se realizó a partir de los datos obtenidos de la cátedra de Física-Química Biológica durante el ciclo lectivo 20II, asignatura que pertenece al Ciclo básico de la carrera de Odontología dependiente de la Universidad Nacional del Nordeste (U.N.N.E.), y corresponde al primer año de la carrera, la cual posee un régimen de cursado semestral.

La materia se desarrolló bajo la modalidad semipresencial; complementando los encuentros presenciales tradicionales con los virtuales, con el objetivo de que el alumno pueda organizar mejor sus tiempos de estudio, promoviendo el aprendizaje autónomo a través de diferentes actividades (trabajos prácticos, auto-evaluaciones, material de estudio, etc.). Además, contó con tutorías permanentes que ofrecieron y permitieron al estudiante disipar sus dudas e inquietudes respecto de la disciplina, interactuar de manera constante con sus tutores y compañeros a través del aula virtual.

La duración del cursado, no presentó modificaciones por tratarse de esta modalidad, este se 
desarrolló durante el segundo semestre del año 2011 .

\section{Características de los Destinatarios}

El cursado de la Asignatura durante dicho semestre, estuvo dedicado a los ingresantes, y recursantes de la materia.

De un total de 208 alumnos inscriptos en la cátedra Física-Química, el 100\% optaron por la modalidad de tutorías Virtuales (pudiendo elegir entre Tutoría Presencial y Virtual).

\section{Particularidades del Aula Virtual}

Las Tutorías Virtuales se llevaron a cabo por intermedio del Aula Virtual (AV) de Física Química Biológica, lo cual implicó la configuración de un $\mathrm{AV}$ en la que se habilitaron secciones solicitadas y acordadas previamente. Para ello se utilizó la Plataforma de U.N.N.E. Virtual perteneciente al Departamento de Educación a Distancia de la Universidad Nacional del Nordeste.

El AV consta de diferentes secciones. Las secciones habilitadas fueron: Inicio, Actividades, Otros Documentos, Calificaciones, Noticias, Sitios, Calendario, Foros, Contactos, Mensajería Interna, Chat, Mis Datos, y Dudas. Estas permitieron la participación de todos los actores: Estudiantes, Coordinador, Tutor, Administrador de la Plataforma y Apoyo Técnico-Pedagógico (estos últimos perfiles pertenecen al Programa UNNE-Virtual).

\section{Funcionamiento del Aula: Secciones}

Actividades: En dicha sección se concentraron, las actividades de auto evaluación. Las mismas consisten en actividades de preguntas y respuestas de opción múltiple, las cuales se cargan en una grilla y es el propio sistema el que corrige e informa al alumno si su actividad está aprobada.

Otros Documentos: En esta sección se publicaron materiales de estudio y de consulta, relacionados con la disciplina.

Calificaciones: Cada módulo contó con la publicación de sus correspondientes calificaciones.

Noticias: en esta parte del aula el alumno pudo informarse de todas las actividades de la cátedra, como así también las fechas de los exámenes parciales y recuperatorios.

Sitios: en este lugar se citaron sitios web donde el alumno pudo acceder para observar demostraciones prácticas.

Contactos: por este medio tuvo a disposición cada alumno las direcciones de correo para comunicarse con sus tutores y sus compañeros.

Calendario: en el se publicaron las actividades que se realizaron mes a mes.

Foros: en esta sección se publicaron temas de debates donde el alumno pudo opinar sobre diferentes cuestiones.

Chat: Por este medio el alumno pudo comunicarse vía directa con su tutor asignado (on line) sin la necesidad de acudir a un correo electrónico.

Mensajería Interna: en este lugar el alumno pudo comunicarse con su tutor, preguntarle dudas y enviarle las actividades requeridas por medio de correos electrónicos y mensajes escritos.

Inicio: por medio de ésta sección se registran el número de veces que se ingresa a la Plataforma.

Mis Datos: en esta sección, tanto alumnos como docentes acceden para completar sus datos de interés como sus fechas de nacimiento, dirección o una simple fotografía ${ }^{9-10}$.

\section{Gráficos ilustrativos de participaciones}

Los datos presentados fueron obtenidos de los registros presentes en la sección Administración de la Plataforma de UNNE Virtual donde el sistema automáticamente registra la participación de todos los participantes de la Plataforma.

A continuación se detallan el numero de participaciones de las secciones mencionadas anteriormente durante los meses de agosto a diciembre.Se observa que los meses de Agosto y Septiembre (gráficos I y 2) hubieron un mayor número de ingresos a las diferentes secciones del Aula virtual, principalmente debido a la falta de familiarización con las actividades y falta de manejo del Aula Virtual. 
Gráfico $\mathbf{N}^{\circ}$ I: Número de participaciones durante el mes de Agosto.

\begin{tabular}{|l|r|}
\hline secciones & \\
\hline actividades & 12740 \\
\hline otros documentos & 8999 \\
\hline calificaciones & 3939 \\
\hline noticias & 5121 \\
\hline sitios & 560 \\
\hline calendario & 749 \\
\hline foros & 204 \\
\hline chat & 224 \\
\hline contactos & 557 \\
\hline mensajeria interna & 4358 \\
\hline inicio & 8182 \\
\hline mis datos & 396 \\
\hline
\end{tabular}

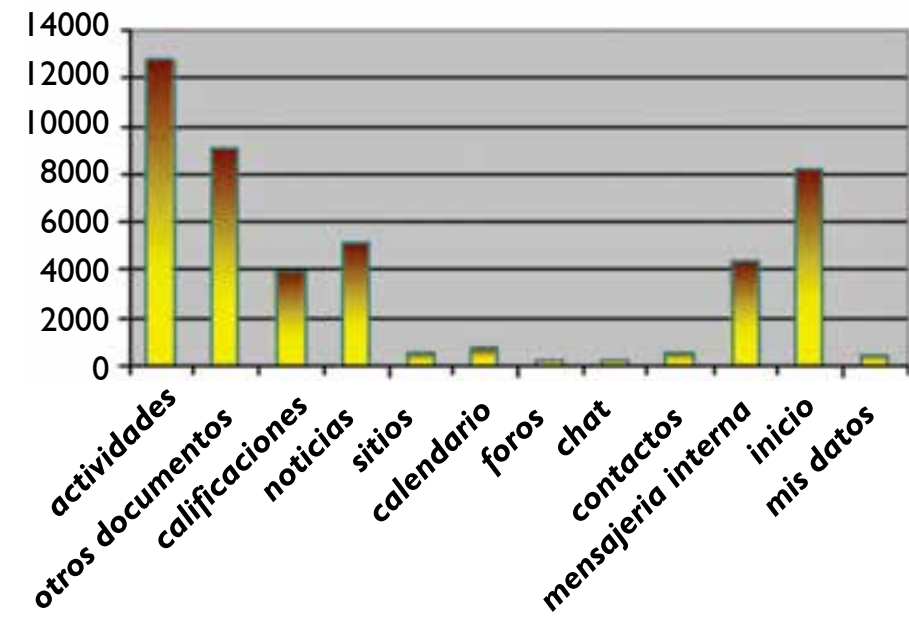

Gráfico N²: Número de participaciones durante el mes de Septiembre.

\begin{tabular}{|l|r|}
\hline secciones & \\
\hline actividades & 12740 \\
\hline otros documentos & 8999 \\
\hline calificaciones & 3939 \\
\hline noticias & 5121 \\
\hline sitios & 560 \\
\hline calendario & 749 \\
\hline foros & 204 \\
\hline chat & 224 \\
\hline contactos & 557 \\
\hline mensajeria interna & 4358 \\
\hline inicio & 8182 \\
\hline mis datos & 396 \\
\hline
\end{tabular}

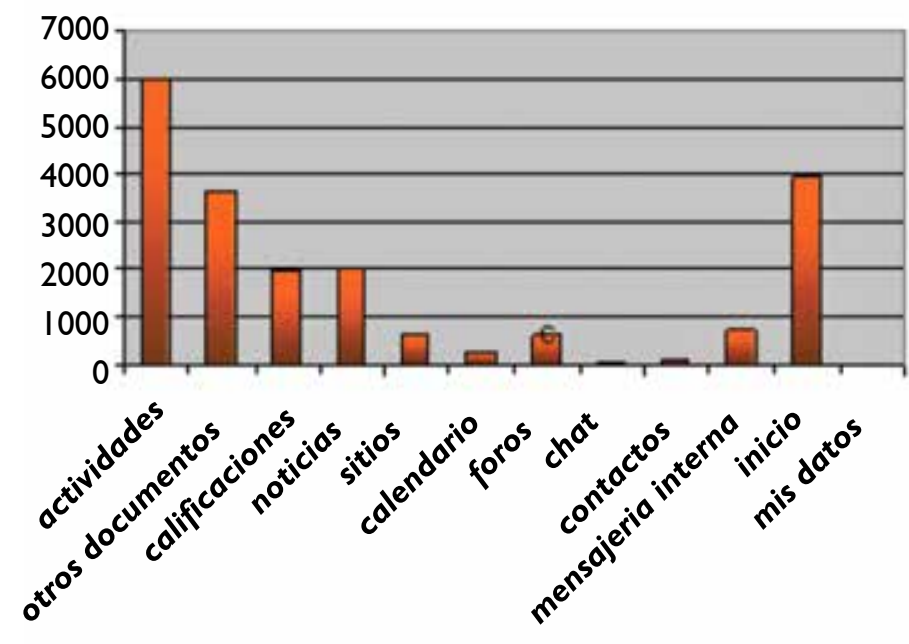

a Serie I 
Gráfico $\mathbf{N}^{\circ}$ 3: Número de participaciones durante el mes de Octubre.

\begin{tabular}{|l|r|}
\hline secciones & \\
\hline actividades & 2546 \\
\hline otros documentos & 3425 \\
\hline calificaciones & 914 \\
\hline noticias & 1348 \\
\hline sitios & 92 \\
\hline calendario & 134 \\
\hline foros & 1800 \\
\hline chat & 19 \\
\hline contactos & 86 \\
\hline mensajeria interna & 1323 \\
\hline Inicio & 3404 \\
\hline mis datos & 15 \\
\hline
\end{tabular}

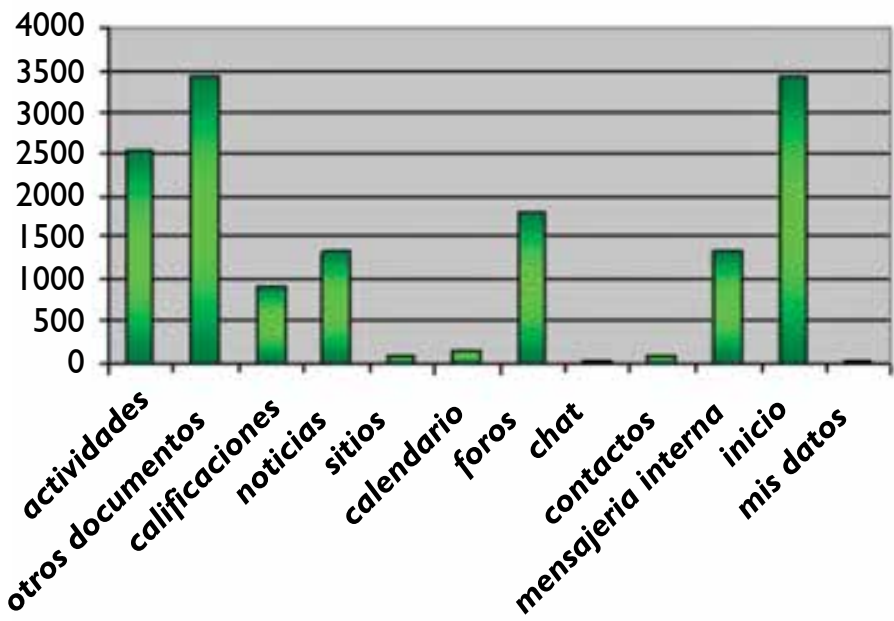

Serie I

Gráfico $\mathbf{N}^{\circ}$ 4: Número de participaciones durante el mes de Noviembre.

\begin{tabular}{|l|r|}
\hline secciones & \\
\hline actividades & 245 \\
\hline otros documentos & 745 \\
\hline calificaciones & 663 \\
\hline noticias & 602 \\
\hline Sitios & 25 \\
\hline calendario & 28 \\
\hline Foros & 72 \\
\hline Chat & 13 \\
\hline contactos & $6 \mathrm{I}$ \\
\hline mensajeria interna & $67 \mathrm{I}$ \\
\hline Inicio & $\mathrm{I} 162$ \\
\hline mis datos & 2 \\
\hline
\end{tabular}

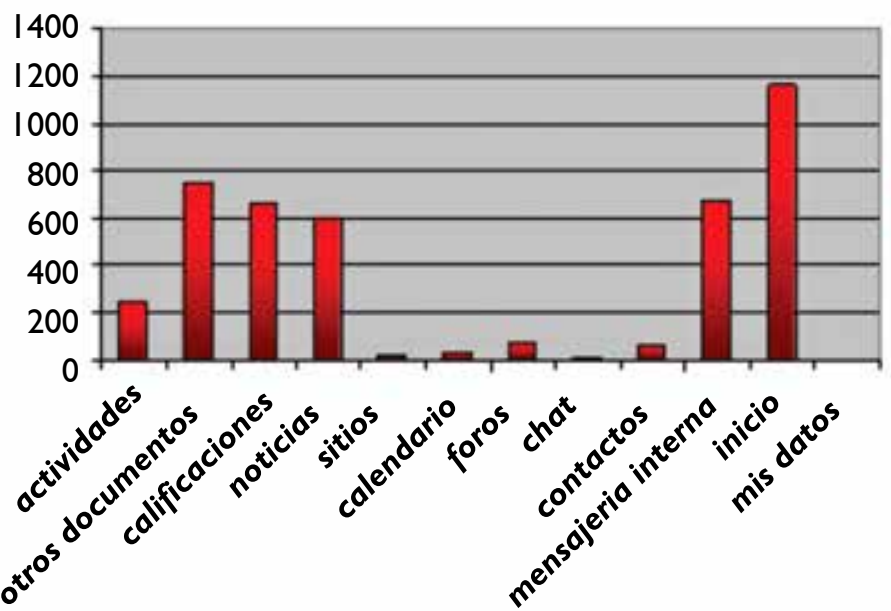

a Serie I 
Gráfico N 5: Número de participaciones durante el mes de Diciembre.

\begin{tabular}{|l|r|}
\hline secciones & \\
\hline actividades & 39 \\
\hline otros documentos & 97 \\
\hline calificaciones & 29 \\
\hline noticias & 58 \\
\hline Sitios & 2 \\
\hline calendario & 10 \\
\hline Foros & 0 \\
\hline Chat & 1 \\
\hline contactos & 1 \\
\hline mensajeria interna & 32 \\
\hline Inicio & 97 \\
\hline mis datos & 0 \\
\hline
\end{tabular}

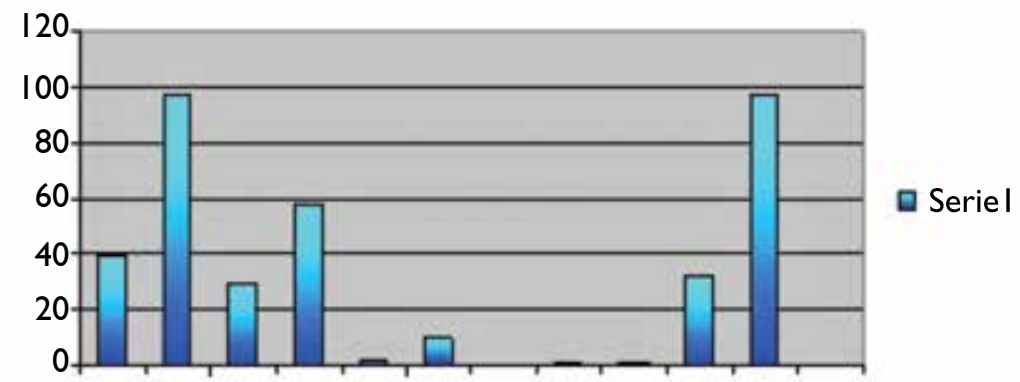

A continuación se detallan la totalidad de los registros de los ingresos durante todo el cursado $20 \mathrm{I}$.

Gráfico $N^{\circ}$ 6: Número total de participaciones de Agosto a Diciembre.

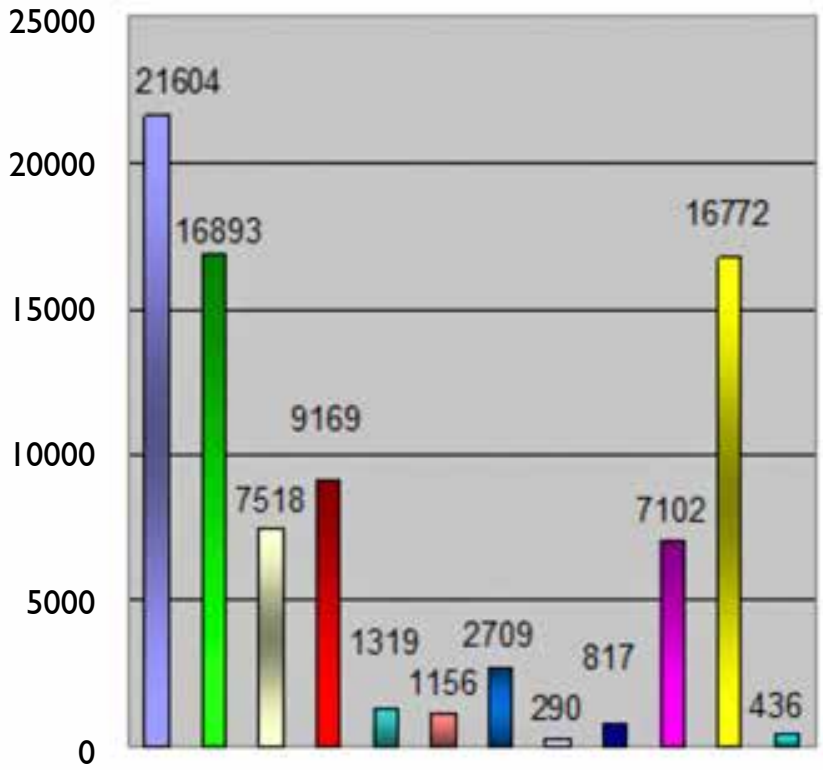

actividades
$\square$ otros documentos
日 calificaciones
noticias
sitios
calendario
foros
$\square$ chat
contactos
$\square$ mensajeria interna
$\square$ inicio
$\square$ mis datos

G actividades

$\square$ otros documentos

ones

ㅁalendario

a foros

日 inicio 
La sección más visitada fue la de Actividades, lo cual demuestra el alto grado de interés de parte de alumno en realizar las actividades solicitadas (21.604 visitas).

La otra sección que captó el mayor interés de parte del cursante fue la de otros documentos, lo cual denota que el alumno descargó el material de estudio y consulta (I6.893 visitas).

La sección inicio indica el número de veces que se inició la sesión lo cual nos demuestra el elevado número de ingresos a ala página (16.772).

Le sigue en grado de importancia la sección noticias lo cual sirve para demostrar que el alumno estaba pendiente de toda la información brindada por la cátedra ( 9.169 visitas)

El número de participaciones en la sección calificaciones demuestra el interés que tenía el cursante en saber el grado de avance y las notas obtenidas en los trabajos de autoevaluación solicitados (7.518 visitas).

La otra sección utilizada en similar grado de importancia fue la de Mensajería interna, la cual da a conocer el grado de comunicación que los alumnos tienen con su tutor (7.102 visitas.)

Gráfico N 7: Porcentajes de participaciones de Agosto a Diciembre.

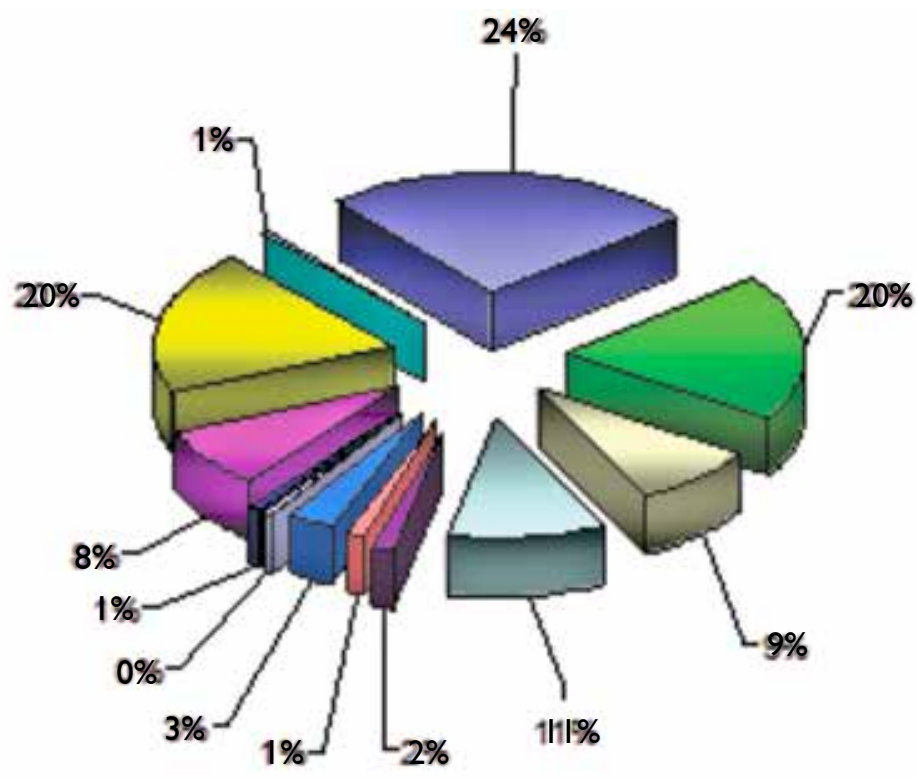

actividades

$\square$ otros documentos

$\square$ calificaciones

$\square$ noticias

a sitios

$\square$ calendario

$\square$ foros

$\square$ chat

contactos

$\square$ mensajeria interna

$\square$ inicio

$\square$ mis datos 
Cumplimiento de las actividades propuestas por la Cátedra por parte de los alumnos.

Otro de los valores importantes a tener en cuenta fue el altísimo número de alumnos que cumplió con todas las actividades propuestas 173 alumnos totalizaron el $100 \%$ de las actividades, 13 cumplieron con el $92 \%, 7$ con el $84 \%, 2$ con el $76 \%, 4$ con el $69 \%$, I con el $61 \%, 3$ con el $53 \%$, 2 con el $46 \%$, I con el $38 \%$ y 2 con el $0 \%$, según los registros obtenidos de la plataforma de U.N.N.E. Virtual.-.

Gráfico N 8: Numero de Alumnos y Porcentaje de Actividades Realizadas.

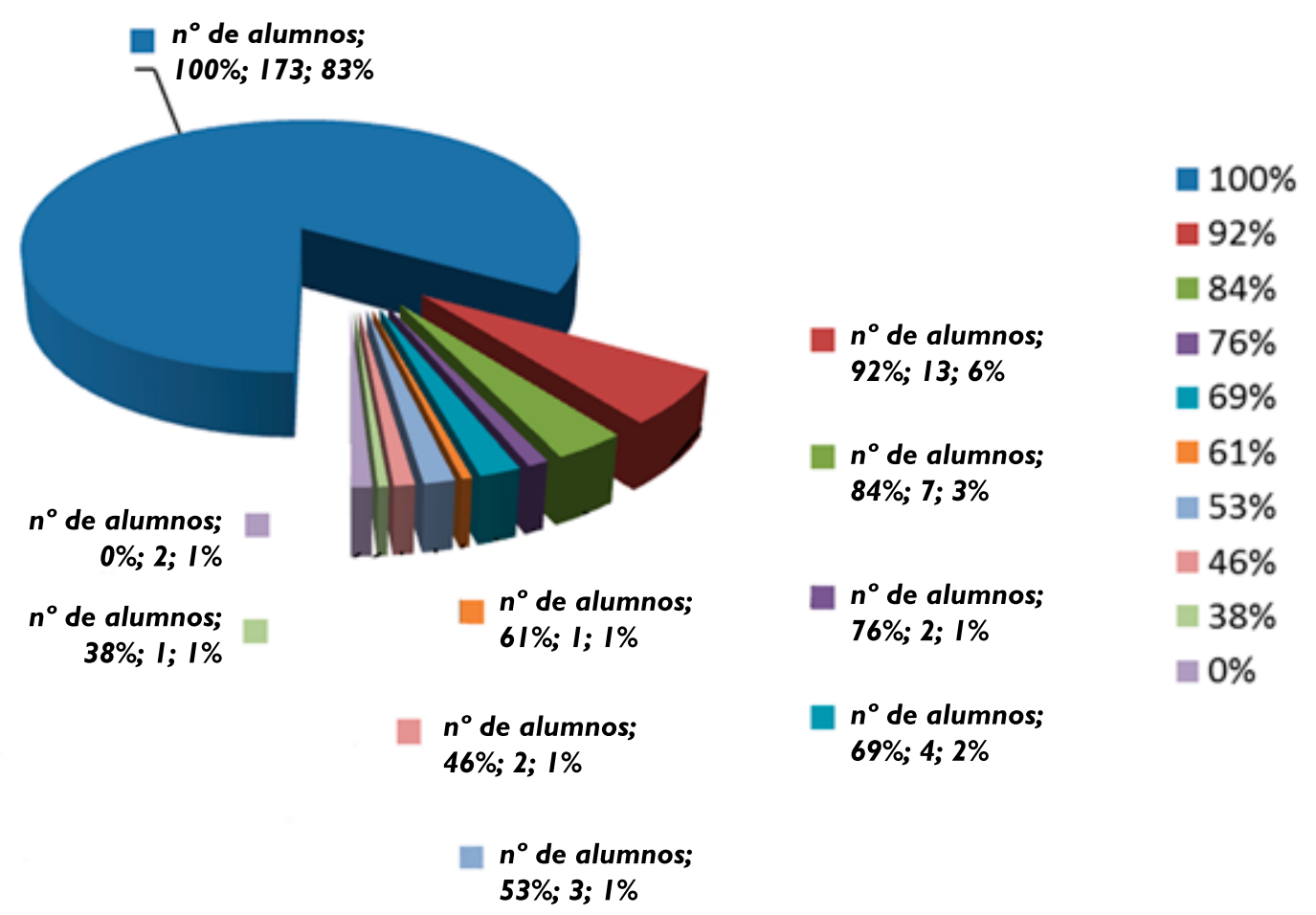

53\%; 3; $1 \%$ 


\section{Conclusión}

El aula Virtual funcionó durante todo el período del cursado con un elevado grado de participación por parte de los alumnos, las secciones actividades y otros documentos fueron las más visitadas, secciones en las cuales se concentraron las actividades de auto evaluación, y se publicaron materiales de estudio y de consulta relacionados con la disciplina.

Teniendo en cuenta los resultados obtenidos podemos considerar que el Aula Virtual es una herramienta adecuada para el complemento de las actividades presenciales llevadas a cabo en la cátedra.

Como reflexión final se puede decir que el Aula Virtual sirvió de gran ayuda, tanto para poder aportar información disciplinar como del planeamiento de actividades de la cátedra además de todos los reconocidos beneficios que logra la Educación a Distancia. El alto grado de participación de los integrantes lo demuestra.

\section{Bibliografía}

I. Castillo López O., Contreras de Villar A., García Ocaña E., Illana Martos A. y cols. "Tutorías virtuales. ¿Realmente tutorías?” XIII Congreso Universitario de Innovación Educativa en las enseñanzas técnicas. Universidad de Cádiz. España. 2005 http://www.eup.ulpgc.es/XIIICUIEET/ Ficheros/Ponencias/2I_SEP/Sala_2/PON-C-04. pdf

2. Germán Moltó; Ana M. Fita; José F. Monserrat y cols. "La tutoría virtual para la autogestión del aprendizaje en las enseñanzas técnicas"-Univest 20II. Universidad Politécnica de Valencia. España. 20I I.- http://dugi-doc.udg.edu/bitstream/ handle/I0256/3779/I I 8.pdf?sequence= I

3. Ricardo Díez, Fernando R Cuzziol., Miguel Acuña, Rolando Juárez. "Las Tutorías Virtuales en la Cátedra de Biofísica: nuevas estrategias y nuevos desafíos. Facultad de Odontología. Universidad Nacional del Nordeste". Trabajo Publicado en Revista de la Facultad de Odontología Universidad Nacional del Nordeste; Corrientes - Argentina. ISSN 1668-7280-2008-Volumen I - $\mathrm{N}^{\circ} 3$ - Pp. 27-36.

4. Reyes Varela, Fernando "La era digital: valor y uso de las nuevas tecnologías educativas". Revista Digital Universitaria. 10 de febrero 2008, Vol. 9, No. 2. [Consultada: 25 de Junio de 2012].
Disponible en Internet: http://www.revista.unam. mx/vol.9/num2/art08/int08.htm ISSN: 1607-6079.

5. Goéry Delacôte. "Enseñar y aprender con nuevos métodos. La revolución cultural de la era electrónica". España: Editorial Gedisa, 1997, p. I20. ISBN 9788474326338

6. Andrea Elizabeth Guzmán Castro. Tutorías virtuales como herramienta complementaria y su efecto sobre las evaluaciones globales en estudiantes universitarios Revista lberoamericana de Educación Nov 2003 [consultada el 26 de Junio de 2012]. Disponible en internet: <bvs.sld.cu/ revistas/ems/vol25_3_II/ems053II.htm>

7. CIP. Centro de Informática Psicopedagógica SRL "¿Que son las aulas virtuales?" Sitio web "Aulas Virtuales" Consultada el 25/06/20I2 URL: http:// www.tizaypc.com/cip-av/av-infol.htm

8. Beatriz Contreras Arroyo. "Internet en la educación" Revista digital Innovación y experiencias educativas. Abril de $2010 \mathrm{~N}^{\circ} 29$ [Consultada: 25 de Junio de 2012]. Disponible en Internet: <http://www.csicsif.es/andalucia/modules/ mod_ense/revista/pdf/Numero_29/BEATRIZ CONTRERAS_ARROYO_OI.pdf $>$ ISSN I988-6047.

9. María Victoria López; Gladys N. Dapozo ; Cristina L. Greiner; María C. Espíndola."Entorno virtual como soporte a la formación de informáticos en una universidad del nordeste argentino" EDUTEC. Revista Electrónica de Tecnología Educativa. Marzo 2012. Núm. 39 [Consultada: 25 de Junio de 2012]. Disponible en Internet: <http://edutec.rediris.es/Revelec2/Revelec39/ pdf/Edutece_39_Lopez_Dapozo_Greiner_ Espindola.pdf > ISSN II 35-9250

10. Juan Manuel Ayllón Díaz-González "El uso de las plataformas de enseñanza virtual para impartir asignaturas jurídicas" REJIE. Revista Jurídica de Investigación e Innovación Educativa. [Consultada: 26 de Junio de 2012]. Disponible en Internet: <http://www.eumed.net/rev/rejie/0I/jmadg.htm> ISSN: 1989-8754

\section{Agradecimientos}

Se agradece al señor Facundo Ledesma por su colaboración en el presente trabajo. 